\title{
Effects of supplemented culture media from solid-state fermented Isaria cicadae on performance, serum biochemical parameters, serum immune indexes, antioxidant capacity and meat quality of broiler chickens
}

\author{
Shaoshuai Liu', Wenjuan Yan², Chang Ma', Yajing Liu', Limin Gong ${ }^{1}$, Crystal Levesque, and Bing Dong ${ }^{1, *}$
}

* Corresponding Author: Bing Dong Tel: +86-131-4619-5659, Fax: +86-10-62733688,

E-mail: dongbing@cau.edu.cn

${ }^{1}$ State Key Laboratory of Animal Nutrition, College of Animal Science and Technology, China Agricultural

University, Beijing 100193, China

2 Zhejiang BioAsia Biomedical Co., Ltd., Pinghu,

Zhejiang, 314200, China

${ }^{3}$ Monogastric Nutrition Department of Animal Science, College of Agriculture and Biological Sciences, South

Dakota State University, Brookings, South Dakota 57007, USA

ORCID

Shaoshuai Liu

https://orcid.org/0000-0003-2092-6700

Wenjuan Yan

https://orcid.org/0000-0001-7696-6934

Chang Ma

https://orcid.org/0000-0002-7075-8883 Yajing Liu

https://orcid.org/0000-0001-9347-7665

Limin Gong

https://orcid.org/0000-0002-0319-4678

Crystal Levesque

https://orcid.org/0000-0003-2978-5852

Bing Dong

https://orcid.org/0000-0002-1150-3493

Submitted Mar 7, 2019; Revised Jun 2, 2019; Accepted Jul 6, 2019
Objective: The objective of this study was to investigate effects of supplementation of culture media from solid-state fermented Isaria cicadae (I. cicadae) on performance, serum biochemical parameters, serum immune indexes, antioxidant capacity and meat quality of broiler chickens.

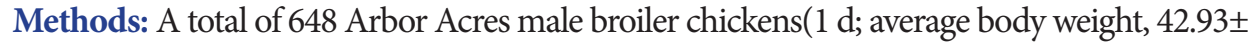
$0.47 \mathrm{~g}$ ) were randomly assigned to 6 treatments, each with six replicates and 18 broiler chickens per replicate. Broiler chickens were fed phase I (d 1 to 21) and phase II (d 22 to 42) diets. The phase I diets were corn and soybean-meal based diets supplemented with $0 \%, 2 \%, 4 \%$, $6 \%, 8 \%$, or $10 \%$ culture media from solid-state fermented I. cicadae respectively. The phase II diets were corn and soybean-meal based diets supplemented with $0 \%, 1.33 \%, 2.67 \%, 4.00 \%$, $5.32 \%$, or $6.67 \%$ culture media from solid-state fermented I. cicadae respectively.

Results: In phase I, the broiler chickens with the supplementation of culture media had increased body weight gain and feed intake (linear and quadratic, $\mathrm{p}<0.05$ ) with increasing inclusion of culture media. The levels of serum total antioxidant capacity (T-AOC), glutathione $(\mathrm{GSH})$ and superoxide dismutase (SOD) increased linearly $(\mathrm{p}<0.05)$. In phase II, levels of serum T-AOC and interleukin-1 $\beta$ increased linearly $(\mathrm{p}<0.05)$, and GSH increased $(\mathrm{p}<0.05)$. In the kidney, GSH and glutathione peroxidase (GSH-Px) concentrations increased (linear and quadratic, $\mathrm{p}<0.05)$ and SOD concentration increased linearly $(\mathrm{p}<0.05)$. Compared to the control, shear force and drip loss of breast muscle decreased (linear and quadratic, $\mathrm{p}<$ 0.05). Drip loss of leg muscle decreased linearly and quadratically $(\mathrm{p}<0.05)$.

Conclusion: Dietary supplementation of culture media from solid-state fermented I.cicadae which was enriched in both wheat and residual bioactive components of I. cicadae enhanced the growth performance of broiler chickens. It also improved body anti-oxidative status and contributed to improve broiler meat quality.

Keywords: Culture Media of Solid-state Fermented Isaria cicadae; Serum Biochemical Parameters; Serum Immune Indexes; Antioxidant Capacity; Meat Quality

\section{INTRODUCTION}

Isaria cicadae (I. cicadae) is a famous and valuable traditional Chinese medicine. It is known as Chan Hua in China and is formed in cicada nymphs infected by Paecilomyces cicadae [1]. I. cicadae has many putative active functions such as: antitumor activity, analgesic activity and amelioration of renal function [2,3]. I. cicadae is an anamorph of Cordyceps cicadae which contains not only nutrients such as proteins, amino acids and various trace elements, but also various bioactive constituents such as sterols, cyclic peptides, nucleosides, and 
polysaccharides [4-6]. As market demand increases, natural supplies of I. cicadae have been widely depleted due to over harvesting, thus artificial culture of I. cicadae by inoculation of I. cicadae on plants (wheat or rice) provides a productive way to obtain large-scale I. cicadae. There are few studies reporting that feeding chickens with I. cicadae as a feed additive can improve immune functions [7] and muscle meat quality [8], while it is unlikely to be popularly applied in animal feed due to the high cost.

In the large scale production of artificial culture of I. cicadae, a large amount of discarded I. cicadae culture media are produced after harvesting the fruiting bodies of I. cicadae for medicinal usage. During the cultivation, mycelia and many bioactive ingredients are secreted into the culture media, which contains many nutrients and active ingredients [9]. Culture media of solid-state fermentation is usually composed of plant (wheat or rice) and residual components of I. cicadae. Measurement of the nutrient composition and active substances of a typical abandoned cultured medium reveals that it contains a considerate percentage of crude protein, crude fat, carbohydrate and gross energy (GE). The medium is also rich in bioactive components such as cordyceptin (adenosine), Dmannitol, N6-(2-hydroxyethyl), polysaccharides and numerous essential amino acids. Compared to the fruiting body of $I$. cicadae, the abandon media contains less protein, fat, fiber and bioactive components but more carbohydrates and GE. It demonstrates the abandon media of solid fermented $I$. cicadae is an excellent feed ingredient for animals.

In this study, we evaluate the effects of supplementation with I. cicadae waste media as a feed ingredient on the growth performance, serum biochemical parameters, serum immune parameters, antioxidant status and meat quality in broiler chickens.

\section{MATERIALS AND METHODS}

\section{Chemical analysis of culture media}

Culture media of I. cicadae was collected once the solid-state fermentation completed. The fermentation process was described briefly as following. The conidia of I. cicadae were collected from agar slants by sterilized distilled water washing. This liquid form of conidia was filtered followed by cultivation in seed culture media at a suitable concentration. Then $5 \mathrm{~mL}$ of the seed culture medium was inoculated to the medium of solid-state fermentation (mixture of $20 \mathrm{~g}$ wheat and $32 \mathrm{~mL}$ basal liquid supplement medium) and allowed to be incubated at $20^{\circ} \mathrm{C}$ for $12 \mathrm{~d}$ in the dark followed by the incubation at $23^{\circ} \mathrm{C}$, $60 \%$ to $70 \%$ relative humidity and $500 \mathrm{~lx} 12 \mathrm{hrs} / \mathrm{d}$ for 12 to $20 \mathrm{~d}$. The basal liquid contained sucrose, peptone, magnesium sulfate and monopotassium phosphate. The fruiting body of I. cicadae was harvested by cutting along the surface of wheat medium. The remaining wheat medium was the cultured medium of solid-state fermented I. cicadae used in this study.

Culture media of solid-state fermented I. cicadae was analyzed for crude protein, crude fat, calcium, and total phosphorus following AOAC 2013. The GE was determined by an Automatic Energy Analyzer (Parr 1281, Moline, IL, USA). AAs except for methionine and tryptophan were measured using ion-exchange chromatography with an Automatic Amino Acid Analyzer (L-8800; Hitachi Automatic Amino Acid Analyzer, Tokyo, Japan) after hydrolyzing with $6 \mathrm{~mol} / \mathrm{L} \mathrm{HCl}$ at $110^{\circ} \mathrm{C}$ for $24 \mathrm{~h}$. Methionine was measured after oxidation with performic acid and subsequent hydrolysis with $\mathrm{HCl}$ while tryptophan was determined after alkaline hydrolysis at $120^{\circ} \mathrm{C}$ for $16 \mathrm{~h}$ (AOAC, 2013) with separation using reverse-phase High performance liquid chromatography (Waters 2690, Milford, MA, USA). Carbohydrates were measured by Sulfuric Acid-UV method [10]. soluble dietary fiber, insoluble dietary fiber and total dietary fiber were analyzed using the Ankom Dietary Fiber Analyzer (Ankom Technology, Macedon, NY, USA) according to a reported modified procedure [11]. Culture media from solid-state fermented I. cicadae was from Zhejiang BioAsia Biomedical Co., Ltd. (Pinghu, Zhejiang, China). Its nutritional contents are shown in Table 1.

\section{Animals and experiment design}

A total of 648 Arbor Acres male broiler chickens (1 d; average body weight, $42.93 \pm 0.47 \mathrm{~g}$ ) were purchased from Arbor Acres Poultry Breeding Company (Beijing, China). On d 1, all birds were randomly assigned to 6 treatments, each with six replicates and 18 broiler chickens per replicate. Broiler chickens were fed phase I ( 1 to 21 ) and phase II (d 22 to 42) diets. The experiment used a corn and soybean-meal based diet which was supplemented with $0 \%, 2 \%, 4 \%, 6 \%, 8 \%$, or $10 \%$ culture medium of solid-state fermented I. cicadae in phase I and $0,1.33 \%, 2.67 \%, 4.00 \%, 5.32 \%$, or $6.67 \%$ in phase II for groups 1 to 6, respectively. All diets were fed in mash form. All nutrients contained in the basal diet met or exceeded the requirements suggested by the Chinese broiler breeding standard (Standard number NY/T33-2004). The composition of the diets is shown in Table 2.

The experiment was conducted at National Feed Engineering Technology Research Centre (Chengde, Hebei, China). All chicks were kept under the same hygienic, managerial and environmental conditions. The temperature in the house was initially at $34^{\circ} \mathrm{C}$ after which the temperature was gradually reduced by $2^{\circ} \mathrm{C}$ a week until it reached $24^{\circ} \mathrm{C}$ and then maintained at this temperature until the end of the 42 -d experiment. All broiler chickens had ad libitum access to feed and water. All chicks were inoculated with inactivated Newcastle Disease Vaccine on d 7 and d 21 and inactivated Infectious Bursal Disease Vaccine on $\mathrm{d} 14$ and $\mathrm{d} 28$. 
Table 1. Nutritional and bioactive components of solid-state fermented Isaria cicadae (cultured medium and fruiting body, respectively)

\begin{tabular}{|c|c|c|}
\hline Items & $\begin{array}{c}\text { Solid-state } \\
\text { cultured medium }\end{array}$ & $\begin{array}{l}\text { Fruiting } \\
\text { body }\end{array}$ \\
\hline \multicolumn{3}{|l|}{ Nutritional components } \\
\hline Gross energy (MJ/kg) & 15.48 & 13.8 \\
\hline Protein (g/100 g) & 15.61 & 30.1 \\
\hline Crude fat $(\mathrm{g} / 100 \mathrm{~g})$ & 1.4 & 5.2 \\
\hline Saturated fatty acids (g/100 g) & 0.3 & 1.31 \\
\hline Unsaturated fatty acids (g/100 g) & 1.4 & 2.11 \\
\hline Carbohydrates (g/100 g) & 70.7 & 26.7 \\
\hline Total fiber $(\mathrm{g} / 100 \mathrm{~g})$ & 6.8 & 27.74 \\
\hline Soluble fiber $(\mathrm{g} / 100 \mathrm{~g})$ & 4.4 & 2.49 \\
\hline Calcium (g/100 g) & 0.11 & 2.1 \\
\hline Total phosphorus (g/100 g) & 0.32 & 1.1 \\
\hline \multicolumn{3}{|l|}{ Bioactive components } \\
\hline D-mannitol (mg/g) & 26.56 & 60 \\
\hline Adenosine (mg/100 g) & 10.8 & 110 \\
\hline N6-(2-hydroxyethyl) (mg/100 g) & 43 & 130 \\
\hline Polysaccharides (mg/g) & 210.44 & 45 \\
\hline \multicolumn{3}{|l|}{ Amino acid contents ( $\mathrm{g} / 100 \mathrm{~g})$} \\
\hline Methionine & 0.17 & 0.22 \\
\hline Cystine & 0.23 & 0.44 \\
\hline Lysine & 0.31 & 0.94 \\
\hline Threonine & 0.44 & 1.09 \\
\hline Tryptophan & 0.13 & 1.53 \\
\hline Arginine & 0.52 & 1.12 \\
\hline Isoleucine & 0.36 & 0.44 \\
\hline Leucine & 0.62 & 1.49 \\
\hline Valine & 0.53 & 0.84 \\
\hline Histidine & 0.19 & 0.39 \\
\hline Phenylalanine & 0.43 & 0.74 \\
\hline Glycine & 0.46 & 1.01 \\
\hline Serine & 0.51 & 1.16 \\
\hline Proline & 1.02 & 1.93 \\
\hline Alanine & 0.485 & 1.54 \\
\hline Aspartic acid & 0.76 & 2.05 \\
\hline Glutamate & 2.14 & 2.31 \\
\hline
\end{tabular}

\section{Collection of performance data}

Feed consumption and body weight of broiler chickens for each replicate after fasting for $12 \mathrm{~h}$ were measured on $\mathrm{d} 21$ and $\mathrm{d} 42$. Body weight gain (BWG), feed intake (FI), and feed conversion ratio (FCR, F:R) were calculated during 1 to $21 \mathrm{~d}, 22$ to $42 \mathrm{~d}$, and 1 to $42 \mathrm{~d}$.

\section{Slaughter and sample collection}

On d 21 and d 42, three broiler chickens whose body weights were close to average body weight were randomly selected from each replicate pen in each treatment and their blood sampled. Serum was collected by centrifugation at $1,500 \times g$ for $10 \mathrm{~min}$ and stored at $-20^{\circ} \mathrm{C}$ for later analysis.

After blood sampling on d 42 , one broiler per pen was bled from the jugular vein and decapitated. After slaughter, liver and kidney were sampled, placed in liquid nitrogen and stored at $-80^{\circ} \mathrm{C}$ for the analysis of antioxidant capacity. Muscles of left breast and leg were sampled for analysis of meat quality.

\section{Assay of serum biochemical parameters \\ The concentrations of serum alanine aminotransferase (ALT), aspartate aminotransferase (AST), total protein (TP), albu- min (ALB), glucose (GLU), total bilirubin (TBiL), alkaline phosphatase (ALP), and uric acid (UA) were measured by corresponding commercial kits (BioSino Bio-technology and Science Inc., Beijing, China) using automatic biochemical analyzer (Hitachi 7160, Hitachi High-Technologies Corpora- tion, Japan).}

\section{Assay of serum immune indexes \\ The concentrations of serum immunoglobulin A (IgA), IgG, IgM, interleukin- $1 \beta$ (IL- $1 \beta)$ and interferon- $\gamma($ IFN- $\gamma$ ) were measured with corresponding commercial kits (Beijing Sino- UK Institute of Biological Technology, Beijing, China) using automatic biochemical analyzer (Hitachi 7160, Hitachi High- Technologies Corporation, Japan).}

\section{Antioxidant capacity}

Serum, liver and kidney were analyzed for total antioxidant capacity (T-AOC), glutathione (GSH), glutathione peroxidase (GSH-Px), superoxide dismutase (SOD) and malondialdehyde (MDA) by commercial kits (Jiancheng Biochemical Reagent Co., Nanjing, China) according to manufacturer's instructions.

\section{Meat quality}

At 45 min postmortem, the $\mathrm{pH}_{45 \min }$ of the breast and leg muscles was detected using an SPK pH meter (pH-star, DK2730, Herlev, Denmark). Meanwhile, the $\mathrm{pH}_{24 \mathrm{~h}}$ was measured at $24 \mathrm{~h}$ postmortem in a chilling room.

Meat color was measured at $45 \mathrm{~min}$ post-mortem using a Minolta chromameter (CR-410, Konica minota, Tokyo, Japan). The values were described as $\mathrm{L}^{*}$ (lightness), $\mathrm{a}^{*}$ (redness), and $\mathrm{b}^{\star}$ (yellowness). Drip loss was measured as described previously $[12,13]$.

After $24 \mathrm{~h}$ post-mortem, samples were used to measure shear force value [14]. Briefly, samples were stored in a vacuum bag, and heated in a water bath at $75^{\circ} \mathrm{C}$ until the internal temperature of the sample reached $70^{\circ} \mathrm{C}$. After cooling under running water, the surface water of samples was removed. Then 10 cylindrical muscle core samples were collected (10 $\mathrm{mm}$ diameter $\times 10 \mathrm{~mm}$ length) from each meat sample, and then were cut parallel to the fiber orientation using a cylindrical cutter. Peak shear force was determined by using a digitaldisplay-muscle tenderness meter (C-LM3B, Tenovo, Harbin, China). 
Table 2. Ingredient composition and nutrient levels of experimental diets (as-fed basis, \%)

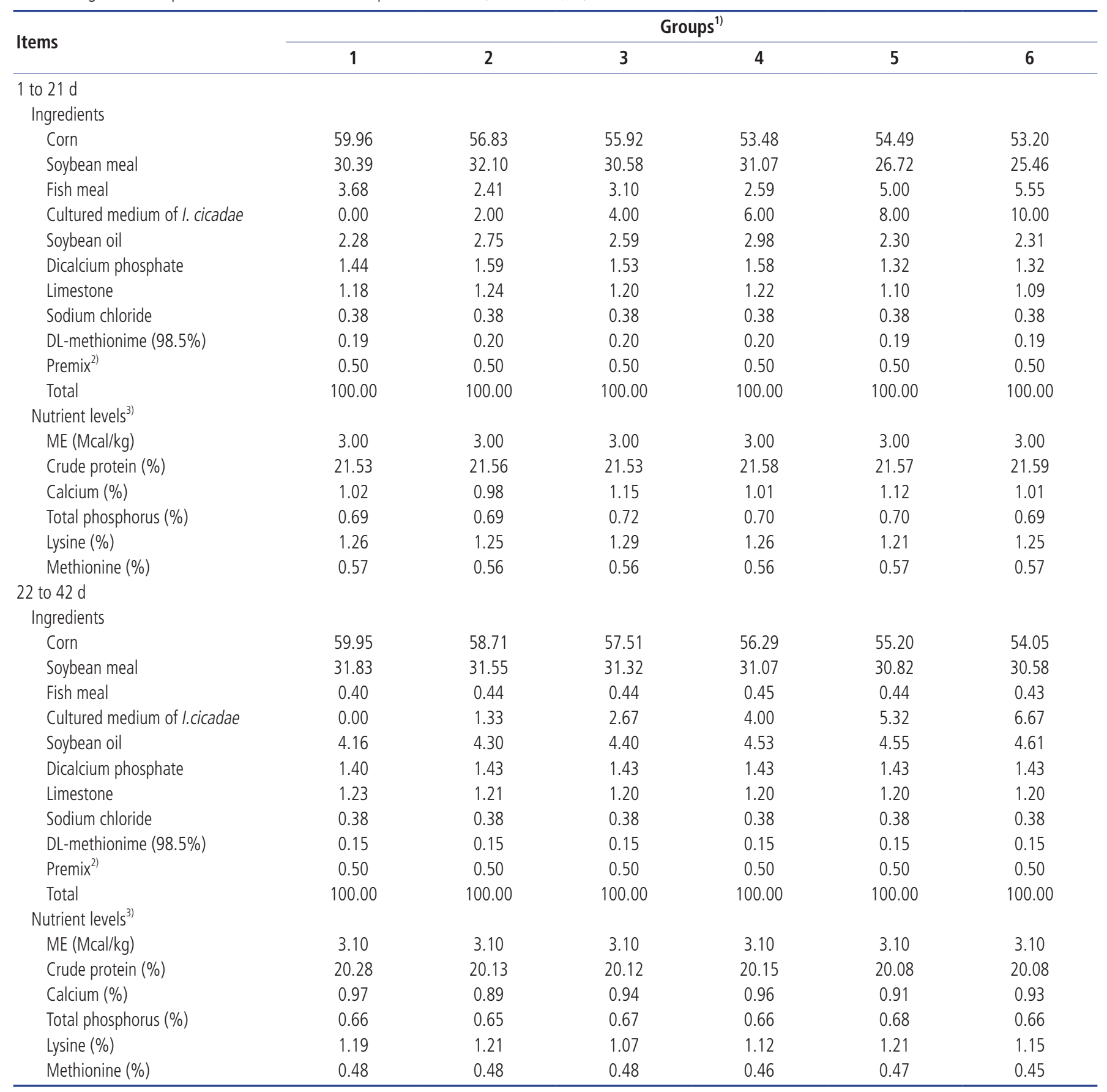

$\mathrm{ME}$, apparent metabolizable energy.

1) The experiment applied a corn and soybean-meal based diet which was supplemented with $0 \%, 2 \%, 4 \%, 6 \%, 8 \%$, or $10 \%$ culture media of solid-state fermented Isaria cicadae in phase I (from d 1 to 21) for groups 1 to 6 respectively, and $0 \%, 1.33 \%, 2.67 \%, 4.00 \%, 5.32 \%$, or $6.67 \%$ in phase II (from d 22 to 42 ) for groups 1 to 6.

2) Premix provided the following per $\mathrm{kg}$ of complete diet: vitamin $A, 10,000 \mathrm{IU}$; vitamin $D_{3}, 2,750 \mathrm{IU}$; vitamin $E_{1}, 30 \mathrm{IU}$; vitamin $\mathrm{K}_{3}, 2 \mathrm{mg}$; vitamin $B_{1}, 1.5 \mathrm{mg}$; vitamin $B_{2}, 6 \mathrm{mg}$;

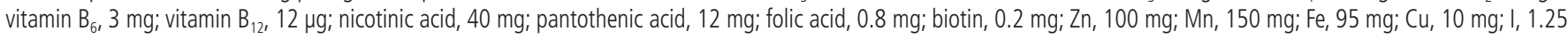
$\mathrm{mg} ; \mathrm{Se}, 0.3 \mathrm{mg}$.

${ }^{3)}$ All nutrient levels except metabolizable energy were analysed.

\section{Statistical analysis}

Data were analyzed using one-way analysis of variance in accordance with the general linear model procedures of SAS 9.4 (SAS Institute Inc., Cary, NC, USA) as a completely ran- domized design including supplementation levels as the fixed effect. Statistical differences among mean values were assessed using Duncan's multiple range test. The pen was deemed as one experimental unit. The model included dietary treatment 
as the only fixed effect. Interactive matrix algebra procedure of SAS was adopted to generate the coefficients of unequally spaced contrasts. Subsequently, the linear and quadratic responses to levels of culture medium from solid-state fermented I. cicadae were assessed by the orthogonal polynomial contrast. Significance level was set at $\mathrm{p}<0.05$.

\section{RESULTS}

\section{Production performance}

The effects of culture media from solid-state fermented $I$. cicadae on the performance of broiler chickens are shown in Table 3. The broiler chickens fed with culture media of $I$. cicadae demonstrated increased BWG and FI as the supplementation increased in phase I (linear and quadratic, $\mathrm{p}<$ 0.05). Supplementing culture medium showed no significant effect on FCR and mortality rate in phase I, nor FI, BWG, FCR and mortality in phase II and the entire phase ( 1 to 42).

\section{Serum biochemical parameters}

No significant difference was observed in the levels of serum ALT, AST, TP, ALB, GLU, TBiL, ALP, and UA in phase I by dietary supplementation of culture media of I. cicadae (Table 4). In phase II, serum AST levels increased quadratically ( $\mathrm{p}<$
0.05). Meanwhile, serum ALP increased linearly $(\mathrm{p}<0.05)$ as the level of culture media increased.

\section{Serum immune indexes}

Supplementation of culture medium from solid-state fermented I. cicadae increased serum IL-1 $\beta$ levels in both phase I and phase II ( $p<0.05$, linearly $\mathrm{p}<0.05)$. Serum IgA in phase I increased $(p<0.05)$ and tended to increase in phase II $(p=$ $0.09)$. Serum IgG tended to increase as the supplementation increased in phase I $(p=0.08)$. Levels of serum IgM and IFN- $\gamma$ were not different in both two phases (Table 5).

\section{Antioxidant capacity}

In phase I, serum T-AOC, GSH, and SOD concentrations all increased linearly $(p<0.05)$ with the increased supplemented level of culture media of I. cicadae, but no differences were observed in serum GSH-Px and MDA concentrations. In phase II, serum T-AOC concentration increased linearly $(\mathrm{p}<0.05)$ with the increased supplemented level of culture media of $I$. cicadae, and serum GSH concentration significantly increased $(\mathrm{p}<0.05)$, but no significant effects on serum GSH-Px, SOD, and MDA levels were observed (Table 6).

In the kidney, GSH and GSH-Px concentrations increased (linear and quadratic, $\mathrm{p}<0.05$ ) with the increased inclusion of culture media. Kidney SOD concentrations increased lin-

Table 3. Effects of graded levels of culture media of solid-state fermented /saria cicadae on growth performance in broiler chickens

\begin{tabular}{|c|c|c|c|c|c|c|c|c|c|c|}
\hline \multirow{2}{*}{ Items } & \multicolumn{6}{|c|}{ Groups ${ }^{1)}$} & \multirow{2}{*}{ SEM } & \multicolumn{3}{|c|}{$p$ value } \\
\hline & 1 & 2 & 3 & 4 & 5 & 6 & & ANOVA & Linear & Quadratic \\
\hline Body weight, initial & 42.9 & 42.9 & 43.1 & 42.9 & 42.9 & 43.0 & 0.16 & 0.97 & 0.94 & 0.92 \\
\hline $21 \mathrm{~d}(\mathrm{~g})$ & $822.9^{c}$ & $839.1^{\text {bc }}$ & $840.7^{\mathrm{bc}}$ & $863.4^{\mathrm{a}}$ & $854.9^{\mathrm{ab}}$ & $836.3^{b c}$ & 4.45 & $<0.01$ & $<0.01$ & $<0.01$ \\
\hline $42 d(g)$ & 2,307 & $2,356.6$ & $2,349.3$ & $2,345.9$ & $2,367.2$ & $2,308.5$ & 17.06 & 0.08 & 0.95 & $<0.01$ \\
\hline \multicolumn{11}{|l|}{1 to $21 \mathrm{~d}$} \\
\hline Feed intake (g/bird) & $1,044^{b}$ & $1,069^{\mathrm{ab}}$ & $1,076^{\mathrm{ab}}$ & $1,094^{\mathrm{a}}$ & $1,083^{\mathrm{ab}}$ & $1,066^{\mathrm{ab}}$ & 9.4 & 0.02 & 0.04 & $<0.01$ \\
\hline Body weight gain (g/bird) & $780^{c}$ & $796^{\mathrm{bc}}$ & $798 b^{c}$ & $820^{a}$ & $812^{a b}$ & $793^{\text {bc }}$ & 4.4 & $<0.01$ & 0.04 & $<0.01$ \\
\hline$F: G$ & 1.34 & 1.34 & 1.35 & 1.33 & 1.33 & 1.34 & 0.01 & 0.92 & $<0.01$ & $<0.01$ \\
\hline Mortality rate (\%) & 2.78 & 2.78 & 1.85 & 0.93 & 0.93 & 0.93 & 1.49 & 0.86 & 0.23 & 0.79 \\
\hline \multicolumn{11}{|l|}{22 to $42 d$} \\
\hline Feed intake (g/bird) & 2,932 & 3,000 & 3,026 & 3,020 & 3,056 & 2,927 & 64.3 & 0.67 & 0.90 & 0.07 \\
\hline Body weight gain (g/bird) & 1,486 & 1,517 & 1,509 & 1,483 & 1,512 & 1,472 & 15.3 & 0.23 & 0.70 & 0.02 \\
\hline$F: G$ & 1.98 & 1.98 & 2.00 & 2.05 & 2.03 & 1.99 & 0.05 & 0.86 & 0.65 & 0.56 \\
\hline Mortality rate (\%) & 0.00 & 0.00 & 0.00 & 0.00 & 0.00 & 0.00 & - & - & - & - \\
\hline \multicolumn{11}{|l|}{1 to $42 \mathrm{~d}$} \\
\hline Feed intake (g/bird) & 3,973 & 4,070 & 4,101 & 4,100 & 4,139 & 3,997 & 61.7 & 0.39 & 0.53 & 0.04 \\
\hline Body weight gain (g/bird) & 2,265 & 2,314 & 2,306 & 2,303 & 2,324 & 2,265 & 16.8 & 0.08 & 0.85 & 0.02 \\
\hline$F: G$ & 1.76 & 1.76 & 1.78 & 1.78 & 1.78 & 1.77 & 0.03 & 0.98 & 0.64 & 0.50 \\
\hline Mortality rate (\%) & 2.78 & 2.78 & 1.85 & 0.93 & 0.93 & 0.93 & 1.49 & 0.86 & 0.23 & 0.79 \\
\hline
\end{tabular}

Values are the means of six replicate pens of 18 birds in each treatment $(n=108)$.

SEM, standard error of the mean; ANOVA, analysis of variance; $F: G$, the ratio of feed to gain.

1) The experiment applied a corn and soybean-meal based diet which was supplemented with $0 \%, 2 \%, 4 \%, 6 \%, 8 \%$, or $10 \%$ culture media of solid-state fermented Isaria cicadae in phase I (from d 1 to 21) for groups 1 to 6 respectively, and $0 \%, 1.33 \%, 2.67 \%, 4.00 \%, 5.32 \%$ or $6.67 \%$ in phase II (from d 22 to 42 ) for groups 1 to 6 .

${ }^{a \cdot c}$ In the same row, values with different letter superscripts mean significant difference $(p<0.05)$, while with the same or no letter superscripts mean no significant difference $(p>0.05)$. 
Table 4. Effects of graded levels of culture media of solid-state fermented Isaria cicadae on serum biochemical parameters in broiler chickens

\begin{tabular}{|c|c|c|c|c|c|c|c|c|c|c|}
\hline \multirow{2}{*}{ Items } & \multicolumn{6}{|c|}{ Groups $^{1)}$} & \multirow{2}{*}{ SEM } & \multicolumn{3}{|c|}{$p$ value } \\
\hline & 1 & 2 & 3 & 4 & 5 & 6 & & ANOVA & Linear & Quadratic \\
\hline \multicolumn{11}{|l|}{$21 d$} \\
\hline AST (U/L) & 138.6 & 151.4 & 140.0 & 148.4 & 177.4 & 147.3 & 18.0 & 0.69 & 0.36 & 0.73 \\
\hline TP (g/L) & 22.82 & 23.22 & 24.03 & 20.55 & 22.53 & 25.32 & 2.31 & 0.79 & 0.70 & 0.41 \\
\hline$A L B(g / L)$ & 9.12 & 9.33 & 9.43 & 8.02 & 9.23 & 9.57 & 0.87 & 0.83 & 0.94 & 0.50 \\
\hline TBiL ( $\mu \mathrm{mol} / \mathrm{L})$ & 8.77 & 10.27 & 9.45 & 8.72 & 8.35 & 9.82 & 0.83 & 0.56 & 0.85 & 0.82 \\
\hline $\operatorname{ALP}(\mu \mathrm{mol} / \mathrm{L})$ & 4,741 & 48,522 & 5,415 & 4,883 & 5,053 & 4,874 & 270.8 & 0.57 & 0.76 & 0.25 \\
\hline UA ( $\mu \mathrm{mol} / \mathrm{L})$ & 254.7 & 229.2 & 238.2 & 173.0 & 194.0 & 234.2 & 31.41 & 0.46 & 0.33 & 0.22 \\
\hline \multicolumn{11}{|l|}{$42 d$} \\
\hline ALT (U/L) & 4.77 & 5.24 & 5.20 & 6.08 & 6.42 & 7.03 & 0.70 & 0.22 & 0.02 & 0.74 \\
\hline TBiL ( $\mu \mathrm{mol} / \mathrm{L})$ & 9.97 & 10.05 & 9.57 & 9.45 & 10.33 & 10.2 & 0.62 & 0.9 & 0.67 & 0.38 \\
\hline $\mathrm{ALP}(\mu \mathrm{mol} / \mathrm{L})$ & $1,930^{b}$ & $1,924^{b}$ & $2,039^{b}$ & $3,512^{\mathrm{a}}$ & $2,185^{b}$ & $3,042^{a}$ & 156.8 & $<0.01$ & $<0.01$ & 0.32 \\
\hline UA ( $\mu \mathrm{mol} / \mathrm{L})$ & 220.33 & 255.67 & 226.33 & 215.67 & 227.00 & 156.33 & 31.33 & 0.38 & 0.09 & 0.17 \\
\hline
\end{tabular}

Values are the means of one broiler selected from each replicate pen in one treatment $(n=6)$.

SEM, standard error of the mean; ANOVA, analysis of variance; ALT, alaine aminotransferase; AST, aspartate aminotransferase; TP, total protein; ALB, albumin; GLU, glucose; TBiL, total bilirubin; ALP, alkaline phosphatase; UA, uric acid.

1) The experiment applied a corn and soybean-meal based diet which was supplemented with $0 \%, 2 \%, 4 \%, 6 \%, 8 \%$, or $10 \%$ culture media of solid-state fermented 1 saria cicadae in phase I (from d 1 to 21) for groups 1 to 6 respectively, and 0\%, 1.33\%, 2.67\%, 4.00\%, 5.32\%, or 6.67\% in phase II (from d 22 to 42 ) for groups 1 to 6 .

$\mathrm{a}, \mathrm{b}$ In the same row, values with different letter superscripts mean significant difference $(p<0.05)$, while with the same or no letter superscripts mean no significant difference $(p>0.05)$.

early $(\mathrm{p}<0.05)$. There were no differences in T-AOC and MDA concentrations among treatments. In the liver, concentrations of GSH-Px and MDA showed slightly differences $(\mathrm{p}=0.05)$ among treatments and showed a quadratic response $(\mathrm{p}<0.05$ and $\mathrm{p}=0.07)$ as the supplementation of culture media increased. There were no differences in T-AOC, GSH, and total SOD concentrations among groups (Table 7).

Table 5. Effects of graded levels of culture media of solid-state fermented Isaria cicadae on serum immune indexes in broiler chickens

\begin{tabular}{|c|c|c|c|c|c|c|c|c|c|c|}
\hline \multirow{2}{*}{ Items } & \multicolumn{6}{|c|}{ Groups ${ }^{1)}$} & \multirow{2}{*}{ SEM } & \multicolumn{3}{|c|}{$p$ value } \\
\hline & 1 & 2 & 3 & 4 & 5 & 6 & & ANOVA & Linear & Quadratic \\
\hline \multicolumn{11}{|l|}{$21 d$} \\
\hline $\lg A(g / L)$ & $0.83^{b}$ & $0.92^{\mathrm{ab}}$ & $1.18^{\mathrm{a}}$ & $1.10^{\mathrm{a}}$ & $0.96^{\mathrm{ab}}$ & $1.06^{\mathrm{a}}$ & 0.05 & 0.04 & 0.13 & 0.49 \\
\hline $\lg G(g / L)$ & 5.63 & 5.68 & 6.36 & 6.09 & 5.97 & 6.07 & 0.12 & 0.08 & 0.26 & 0.51 \\
\hline $\lg M(g / L)$ & 0.74 & 0.75 & 0.93 & 0.82 & 0.71 & 0.73 & 0.05 & 0.31 & 0.36 & 0.16 \\
\hline$\| \mathrm{L}-1 \beta(\mathrm{pg} / \mathrm{mL})$ & $47.54^{b}$ & $50.53^{\mathrm{ab}}$ & $51.61^{\mathrm{ab}}$ & $54.14^{\mathrm{a}}$ & $54.77^{\mathrm{a}}$ & $57.50^{\mathrm{a}}$ & 1.01 & 0.03 & $<0.01$ & 0.46 \\
\hline IFN- $\gamma(p g / m L)$ & 17.23 & 17.98 & 19.56 & 19.85 & 18.39 & 19.01 & 0.28 & 0.09 & 0.36 & 0.05 \\
\hline \multicolumn{11}{|l|}{$42 d$} \\
\hline $\lg A(g / L)$ & 1.16 & 1.16 & 1.22 & 1.10 & 1.18 & 1.15 & 0.05 & 0.09 & 0.22 & 0.18 \\
\hline $\lg G(g / L)$ & 7.31 & 7.21 & 7.35 & 7.48 & 7.18 & 7.46 & 1.50 & 0.26 & 0.18 & 0.24 \\
\hline $\lg M(g / L)$ & 0.89 & 0.86 & 0.81 & 0.93 & 1.21 & 0.99 & 0.05 & 0.20 & 0.19 & 0.25 \\
\hline IL-1 $\beta(p g / m L)$ & $58.13^{b}$ & $59.45^{b}$ & $60.13^{b}$ & $61.96^{\mathrm{ab}}$ & $72.51^{\mathrm{a}}$ & $72.49^{a}$ & 2.25 & $<0.01$ & $<0.01$ & 0.35 \\
\hline IFN- $\gamma(\mathrm{pg} / \mathrm{mL})$ & 17.55 & 17.61 & 17.32 & 18.63 & 18.21 & 17.89 & 0.52 & 0.35 & 0.48 & 0.04 \\
\hline
\end{tabular}

Values are the means of three broiler chickens from each replicate pen in one treatment $(n=18)$.

SEM, standard error of the mean; ANOVA, analysis of variance; Ig, immunoglobulin; IL-1 $\beta$, interleukin-1 $\beta$; IFN- $\gamma$, interferon- $\gamma$.

1) The experiment applied a corn and soybean-meal based diet which was supplemented with $0 \%, 2 \%, 4 \%, 6 \%, 8 \%$, or $10 \%$ culture media of solid-state fermented Isaria cicadae in phase I (from d 1 to 21) for groups 1 to 6 respectively, and $0 \%, 1.33 \%, 2.67 \%, 4.00 \%, 5.32 \%$, or $6.67 \%$ in phase II (from d 22 to 42 ) for groups 1 to 6 .

${ }_{a, b}$ In the same row, values with different letter superscripts mean significant difference $(p<0.05)$, while with the same or no letter superscripts mean no significant difference $(p>0.05)$. 
Table 6. Effects of graded levels of culture media of solid-state fermented Isaria cicadae on serum antioxidant capacity in broiler chickens

\begin{tabular}{|c|c|c|c|c|c|c|c|c|c|c|}
\hline \multirow{2}{*}{ Items } & \multicolumn{6}{|c|}{ Groups $^{1)}$} & \multirow{2}{*}{ SEM } & \multicolumn{3}{|c|}{$p$ value } \\
\hline & 1 & 2 & 3 & 4 & 5 & 6 & & ANOVA & Linear & Quadratic \\
\hline \multicolumn{11}{|l|}{$21 d$} \\
\hline T-AOC (U/mL) & $3.74^{d}$ & $4.56^{\text {cd }}$ & $5.17^{b c}$ & $5.28^{b c}$ & $6.80^{\mathrm{ab}}$ & $7.91^{\mathrm{a}}$ & 0.36 & $<0.01$ & $<0.01$ & 0.34 \\
\hline $\mathrm{GSH}(\mu \mathrm{mol} / \mathrm{L})$ & $9.31^{c}$ & $9.12^{c}$ & $10.51^{b c}$ & $11.41^{\mathrm{ab}}$ & $13.85^{\mathrm{a}}$ & $12.98^{\mathrm{a}}$ & 0.58 & $<0.01$ & $<0.01$ & 0.82 \\
\hline GSH-Px (U/mL) & 776.14 & 722.75 & 715.67 & 733.51 & 702.82 & 701.23 & 26.54 & 0.12 & 0.02 & 0.46 \\
\hline T-SOD (U/mg) & $320.01^{b}$ & $319.32^{b}$ & $370.22^{a}$ & $379.90^{\mathrm{a}}$ & $398.61^{a}$ & $390.76^{a}$ & 13.48 & $<0.01$ & $<0.01$ & 0.18 \\
\hline MDA (nmol/mL) & 3.85 & 3.59 & 3.42 & 3.97 & 4.12 & 3.65 & 0.39 & 0.24 & 0.36 & 0.17 \\
\hline \multicolumn{11}{|l|}{$42 d$} \\
\hline $\mathrm{T}-\mathrm{AOC}(\mathrm{U} / \mathrm{mL})$ & $3.88 b^{c}$ & $4.34^{\mathrm{ab}}$ & $3.59^{c}$ & $5.45^{a}$ & $5.31^{\mathrm{a}}$ & $5.74^{\mathrm{a}}$ & 0.35 & $<0.01$ & $<0.01$ & 0.59 \\
\hline GSH $(\mu \mathrm{mol} / \mathrm{L})$ & $6.01^{b}$ & $8.23^{\mathrm{a}}$ & $9.92^{\mathrm{a}}$ & $8.54^{\mathrm{a}}$ & $8.87^{\mathrm{a}}$ & $10.82^{\mathrm{a}}$ & 0.53 & $<0.01$ & 0.84 & 0.39 \\
\hline GSH-Px (U/mL) & 700.32 & 747.18 & 728.54 & 734.87 & 732.54 & 786.60 & 31.03 & 0.89 & 0.63 & 0.87 \\
\hline T-SOD (U/mg) & 346.65 & 373.23 & 364.18 & 371.20 & 370.19 & 369.51 & 17.52 & 0.83 & 0.69 & 0.58 \\
\hline $\mathrm{MDA}(\mathrm{nmol} / \mathrm{mL})$ & 3.52 & 3.75 & 3.38 & 3.50 & 3.19 & 3.82 & 0.62 & 0.99 & 0.68 & 0.71 \\
\hline
\end{tabular}

Values are the means of three broiler chickens from each replicate pen in one treatment $(n=18)$.

SEM, standard error of the mean; ANOVA, analysis of variance; T-AOC, total antioxidant capacity; GSH, glutathione; GSH-Px, glutathione peroxidase; T-SOD, total superoxide dismutase; MDA, malondialdehyde.

${ }^{1)}$ The experiment applied a corn and soybean-meal based diet which was supplemented with $0 \%, 2 \%, 4 \%, 6 \%, 8 \%$, or $10 \%$ culture media of solid-state fermented Isaria cicadae in phase I (from d 1 to 21) for groups 1 to 6 respectively, and 0\%, 1.33\%, 2.67\%, 4.00\%, 5.32\%, or 6.67\% in phase II (from d 22 to 42 ) for groups 1 to 6 .

${ }^{a-d}$ In the same row, values with different letter superscripts mean significant difference $(p<0.05)$, while with the same or no letter superscripts mean no significant difference $(p>0.05)$.

\section{Meat quality}

$\mathrm{a}^{*}$ and $\mathrm{pH}_{45 \mathrm{~min}}$ values of the breast muscle tended to decrease quadratically $(\mathrm{p}<0.05)$ (Table 8$)$. The shear force and drip loss decreased (linear and quadratic, $\mathrm{p}<0.05$ ) as the culture medium supplementation increased. There was no significant difference in the values of $\mathrm{L}^{*}, \mathrm{~b}^{*}$, and $\mathrm{pH} 24 \mathrm{~h}$. In the leg muscle, drip loss decreased (linear and quadratic, $\mathrm{p}<0.05$ ), but supplementing diets with different levels of culture media of solid- state fermented I. cicadae did not alter the values of $\mathrm{L}^{*}, \mathrm{a}^{*}, \mathrm{~b}^{*}$, $\mathrm{pH}_{45 \min }, \mathrm{pH}_{24 \mathrm{~h}}$, and shear force significantly.

\section{DISCUSSION}

Intensive selection for husbandry animals has concentrated on growth rather than meat quality. In China, city people prefer to consume village raised native broiler chickens. These

Table 7. Effects of graded levels of culture media of solid-state fermented Isaria cicadae on tissues antioxidant capacity in broiler chickens

\begin{tabular}{|c|c|c|c|c|c|c|c|c|c|c|}
\hline \multirow{2}{*}{ Items } & \multicolumn{6}{|c|}{ Groups ${ }^{1)}$} & \multirow{2}{*}{ SEM } & \multicolumn{3}{|c|}{$p$ value } \\
\hline & 1 & 2 & 3 & 4 & 5 & 6 & & ANOVA & Linear & Quadratic \\
\hline \multicolumn{11}{|l|}{ Kidney } \\
\hline $\mathrm{T}-\mathrm{AOC}(\mathrm{U} / \mathrm{mg})$ & 2.06 & 2.09 & 2.03 & 1.83 & 2.26 & 2.14 & 0.19 & 0.73 & 0.67 & 0.50 \\
\hline GSH $(\mu \mathrm{mol} / \mathrm{g})$ & $5.44^{c}$ & $5.43^{c}$ & $6.20^{c}$ & $6.13^{c}$ & $8.49^{b}$ & $10.67^{a}$ & 0.49 & $<0.01$ & $<0.01$ & $<0.01$ \\
\hline GSH-Px (U/mg) & $547.66^{c}$ & $696.71^{b c}$ & $816.95^{a b}$ & $834.78^{a b}$ & $875.62^{\mathrm{a}}$ & $885.21^{\mathrm{a}}$ & 41.54 & $<0.01$ & $<0.01$ & 0.01 \\
\hline T-SOD, (U/mg) & $317.18^{b}$ & $324.23^{b}$ & $369.67^{a}$ & $374.30^{\mathrm{a}}$ & $389.61^{\mathrm{a}}$ & $384.86^{\mathrm{a}}$ & $<0.01$ & 0.01 & $<0.01$ & 0.19 \\
\hline MDA (nmol/mL) & 2.16 & 1.95 & 1.76 & 1.58 & 1.46 & 2.04 & 0.21 & 0.16 & 0.04 & 1.30 \\
\hline \multicolumn{11}{|l|}{ Liver } \\
\hline T-AOC (U/mg) & 1.69 & 1.76 & 1.80 & 1.81 & 1.69 & 1.78 & 0.08 & 0.83 & 0.75 & 0.51 \\
\hline $\mathrm{GSH}(\mu \mathrm{mol} / \mathrm{g})$ & 5.63 & 5.86 & 4.73 & 4.94 & 4.97 & 4.29 & 0.55 & 0.36 & 0.05 & 0.99 \\
\hline GSH-Px (U/mg) & $847.94^{\mathrm{ab}}$ & $827.28^{\mathrm{ab}}$ & $773.45^{b}$ & $901.51 a$ & $901.17^{\mathrm{a}}$ & $876.94^{\mathrm{a}}$ & 30.69 & 0.05 & 0.33 & 0.03 \\
\hline T-SOD (U/mg) & 362.61 & 375.94 & 348.78 & 365.95 & 367.10 & 377.46 & 17.67 & 0.88 & 0.66 & 0.54 \\
\hline MDA (nmol/mL) & $2.16^{\mathrm{ab}}$ & $2.17^{\mathrm{ab}}$ & $2.79^{\mathrm{a}}$ & $2.37^{\mathrm{ab}}$ & $2.08^{b}$ & $2.28^{\mathrm{ab}}$ & 0.16 & 0.05 & 0.94 & 0.07 \\
\hline
\end{tabular}

Values are the means of one broiler selected from each replicate pen in one treatment $(n=6)$.

SEM, standard error of the mean; ANOVA, analysis of variance; T-AOC, total antioxidant capacity; GSH, glutathione; GSH-Px, glutathione peroxidase; T-SOD, total superoxide dismutase; MDA, malondialdehyde.

1) The experiment applied a corn and soybean-meal based diet which was supplemented with $0 \%, 2 \%, 4 \%, 6 \%, 8 \%$, or $10 \%$ culture media of solid-state fermented 1 saria cicadae in phase I (from d 1 to 21) for groups 1 to 6 respectively, and $0 \%, 1.33 \%, 2.67 \%, 4.00 \%, 5.32 \%$, or $6.67 \%$ in phase II (from d 22 to 42 ) for groups 1 to 6 .

${ }^{a \cdot c}$ In the same row, values with different letter superscripts mean significant difference $(p<0.05)$, while with the same or no letter superscripts mean no significant difference $(p>0.05)$. 
Table 8. Effects of graded levels of culture media of solid-state fermented Isaria cicadae on meat quality in broiler chickens

\begin{tabular}{|c|c|c|c|c|c|c|c|c|c|c|}
\hline \multirow{2}{*}{ (tems ${ }^{1)}$} & \multicolumn{6}{|c|}{ Groups $^{2)}$} & \multirow{2}{*}{ SEM } & \multicolumn{3}{|c|}{$p$ value } \\
\hline & 1 & 2 & 3 & 4 & 5 & 6 & & ANOVA & Linear & Quadratic \\
\hline \multicolumn{11}{|l|}{ Breast muscle } \\
\hline$L^{*}$ & 49.73 & 51.06 & 51.80 & 52.32 & 51.81 & 50.81 & 1.10 & 0.62 & 0.24 & 0.03 \\
\hline$a^{*}$ & $12.80^{b}$ & $12.87^{b}$ & $13.37^{b}$ & $14.77^{\mathrm{ab}}$ & $16.04^{\mathrm{a}}$ & $13.86^{b}$ & 0.71 & 0.02 & 0.33 & 0.03 \\
\hline$b^{*}$ & 10.54 & 8.87 & 10.62 & 9.72 & 11.01 & 10.03 & 0.69 & 0.33 & 0.58 & 0.79 \\
\hline $\mathrm{pH}_{45 \min }$ & $6.96^{\mathrm{a}}$ & $6.63^{b}$ & $6.61^{b}$ & $6.77^{\mathrm{ab}}$ & $6.80^{\mathrm{ab}}$ & $6.76^{\mathrm{ab}}$ & 0.08 & 0.05 & 0.67 & 0.04 \\
\hline $\mathrm{pH}_{24 \mathrm{~h}}$ & 6.23 & 6.22 & 6.22 & 6.28 & 6.30 & 6.34 & 0.05 & 0.42 & 0.07 & 0.44 \\
\hline Shear force (N) & $16.46^{\mathrm{a}}$ & $18.29^{a}$ & $12.35^{b}$ & $12.69^{b}$ & $12.22^{b}$ & $15.47^{\mathrm{ab}}$ & 1.06 & $<0.01$ & 0.01 & $<0.01$ \\
\hline Drip loss\% & $11.20^{\mathrm{a}}$ & $8.56^{\mathrm{ab}}$ & $6.54^{b}$ & $7.53^{b}$ & $5.43^{b}$ & $6.51^{b}$ & 0.77 & $<0.01$ & $<0.01$ & 0.01 \\
\hline \multicolumn{11}{|l|}{ Leg muscle } \\
\hline$L^{*}$ & 48.12 & 50.00 & 48.42 & 47.48 & 47.77 & 47.62 & 0.98 & 0.50 & 0.54 & 0.92 \\
\hline$a^{*}$ & 4.34 & 3.92 & 3.34 & 4.52 & 4.99 & 3.72 & 0.58 & 0.41 & 0.83 & 0.57 \\
\hline$b^{*}$ & 11.17 & 9.78 & 8.31 & 8.90 & 9.25 & 8.65 & 0.65 & 0.05 & 0.06 & 0.10 \\
\hline $\mathrm{pH}_{45 \min }$ & 6.78 & 6.70 & 6.56 & 6.73 & 7.07 & 6.82 & 0.15 & 0.32 & 0.20 & 0.84 \\
\hline $\mathrm{pH}_{24 \mathrm{~h}}$ & 6.62 & 6.60 & 6.68 & 6.67 & 6.60 & 6.52 & 0.06 & 0.59 & 0.22 & 0.18 \\
\hline Shear force (N) & 12.91 & 13.83 & 12.26 & 12.91 & 12.26 & 16.08 & 1.06 & 0.14 & 0.21 & 0.08 \\
\hline Drip loss\% & $12.51^{\mathrm{a}}$ & $8.17^{\mathrm{ab}}$ & $5.31^{b}$ & $7.66^{b}$ & $5.13^{b}$ & $5.30^{b}$ & 1.06 & $<0.01$ & $<0.01$ & 0.02 \\
\hline
\end{tabular}

Values are the means of one broiler selected from each replicate pen in one treatment $(n=6)$.

SEM, standard error of the mean; ANOVA, analysis of variance.

1) $L^{*}, a^{*}$ and $b^{*}$ are indicators of meat color.

${ }^{2)}$ The experiment applied a corn and soybean-meal based diet which was supplemented with $0,2 \%, 4 \%, 6 \%, 8 \%$ or $10 \%$ culture media of solid-state fermented Isaria cicadae in phase I (from d 1 to 21) for groups 1 to 6 respectively, and 0, 1.33\%, 2.67\%, 4.00\%, 5.32\%, or 6.67\% in phase II (from d 22 to 42 ) for groups 1 to 6 .

${ }^{a, b}$ In the same row, values with different letter superscripts mean significant difference $(p<0.05)$, while with the same or no letter superscripts mean no significant difference $(p>0.05)$.

broiler chickens take longer to finish before going to market thus have more flavor in their meat. Tibet village broiler chickens uncaged raised in Tibetic plateau are increasingly commercially popular because these broiler chickens are Cordyceps-eating and better tasting. Few studies feeding cordyceps in diets to chickens have been conducted, which demonstrate improved growth performance and deposit of meat nutrients. But feeding chickens with cordyceps is not realistic because of its high cost.

Culture media from solid-state fermented I. cicadae contains both plant stoma, usually wheat or rice, and residual bioactive components of I. cicadae. This makes the culture media potentially a good candidate as an ingredient for broiler feed. In this study, supplementation of culture media from solid-state fermented I. cicadae to broiler chickens, compared to the un-supplemented group, resulted in an increased BWG in phase I ( 1 to 21 ), improved antioxidant status and meat quality with increasing inclusion of the media.

Many plants and fungi can simultaneously improve the body antioxidant status and enhance immune responses. This is probably due to the natural polysaccharides in these herbs [15]. Polysaccharides in Cordyceps are water soluble (CPS-2) [16]. Its predicted structure consists of a repeating unit of $\alpha$-(1,4)-D-glucose and $\alpha-(1,3)-D$-mannose, branched with $\alpha$-(1,4,6)-D-glucose [16]. A Cordyceps polysaccharide is proved to protect neurons from free radicals [17]. In this study, sup- plementation of culture medium from fermented I. cicadae improved antioxidant status marked by increased serum and tissue T-AOC, GSH, GSH-Px levels. This contributed improved meat quality of breast and leg muscles which was reflected by the parameters of meat postmortem $\mathrm{pH}$, water holding capacity, drip loss, meat color, and shear force. The culture media from fermented I. cicadae containing residual bioactive components of I. cicadae, significantly improved meat shear force (breast muscle) and drip loss (breast muscle and leg muscle). Oxidation status is closely related to meat water holding capacity because the cell membrane is composed of unsaturated fatty acids. Oxidation of these fatty acids, in particular, disrupts the membrane structure and fluidity. When the membrane phospholipids are oxidized, cell permeability is altered leading to decreased muscle water-holding capacity. Low drip loss means high meat water holding capacity and high water holding capacity can bring advantages in meat processing and meat appearance for consumers [18]. To protect against oxidative injuries, body anti-oxidative system consisting of anti-oxidative enzymes: SOD, GSH-Px, and catalase which catalyze dismutation of superoxide anions to hydrogen peroxide, the degradation of $\mathrm{H}_{2} \mathrm{O}_{2}$ and hydroperoxides originating from unsaturated fatty acids at the expense of reduced glutathione, and convert $\mathrm{H}_{2} \mathrm{O}_{2}$ into water [19]. There are numerous components showing anti-oxidation activity in I. cicadae, like cordycepic acid (D-mannitol) [20], 
cordycepin (3'-deoxyadenosine) [21-23] and cordyceps polysaccharide $[24,25]$. Others reported antioxidant compounds like isoflavone [26], selenium [27], arginine [28], resveratrol [29], and vitamin E [30] can also be found in I. cidadae. These components all contributed to improve the quality of the broiler meat as antioxidants. This is important for producing competitive meat product for market.

Meat color is reflected by the lightness, redness and yellowness $\left(L^{*}, a^{*}\right.$, and $\left.b^{*}\right)$. Previous reports revealed that muscle fiber type was closely related to meat color. The muscles enriched in mitochondria are slow-oxidative type I red muscles like leg muscle which contains more myoglobin, while the muscles active in glycolytic types are white muscles like breast muscle. Physical training $[31,32]$ and some nutrient factors [29] are reported to change muscle type. In this study, we did observe higher redness value of breast muscle which is affected by muscle myoglobin contents [33] of broiler chickens by feeding cultured medium of fermented I. cicadae to broiler chickens.

Fungi polysaccharides have activities beyond being as antioxidants. A selenium polysaccharide from Cordyceps mycelium showed significant antioxidant activity in vivo in mice, thus it suggested uses as a potential antioxidant enhancing adaptive immune response [34]. Polysaccharide purified from fermentation medium of cordyceps showed stimulation effects on macrophages [35,36] and mononuclear cells [37]. Other bioactive components, cordycepin, cordycepic acid, myriocin and beauvericin in I. cicadae, are reported to inhibit the growth of carcinoma cells [38]. Cordycepin resists the growth of hepatocarcinoma via PI3K/Akt/mTOR and Nrf2/HO-1/NF- $\kappa$ B pathway [39]. In this study, feeding broiler chickens with culture media from solid-state fermented $I$. cicadae in diets increased serum IL-1 $\beta$ levels during phase I (d 1 to 21 ) and phase II (d 22 to 42 ), suggesting it promoted cellular immune responses. The enhanced immune system likely enabled the spared energy from immune defense to be used for growth thus increased BWG of broiler chickens. IL- $1 \beta$ is a member of interleukin family of cytokines. It can trigger complex reactions of combinatorial phosphorylation and ubiquitination activating nuclear factor $\mathrm{kb}$ signaling and JNK to induce the expression of IL-1 target genes (IL-6, IL-8, NF- $\kappa B$ inhibitory protein [Iк-B]) [40]. Macrophages and monocytes, as the sentinel cells of the innate immune system, are the major source of IL- $1 \beta$. Other immune relevant cells such as epithelial cells, endothelial cells and fibroblasts can also produce IL-1 $\beta$ [41-43]. The main function of IL- $1 \beta$ is to control proinflammatory reactions in response to tissue injury by bacteria or virus [44-46].

\section{CONCLUSION}

Taken together, dietary supplementation of culture media from solid-state fermented I. cicadae which was enriched in both plant and residual bioactive components of I. cicadae enhanced the growth performance of broiler chickens. It also improved body anti-oxidative status and contributed to improve shear force and drip loss of broiler leg and breast muscles. Culture media from solid-state fermented I. cicadae could be a potential ingredient for broiler feed.

\section{CONFLICT OF INTEREST}

We certify that there is no conflict of interest with any financial organization regarding the material discussed in the manuscript. Yan W is an employee of Zhejiang BioAsia Biomedical Co., Ltd..

\section{REFERENCES}

1. He L, Shi W, Liu X, Zhao X, Zhang Z. Anticancer action and mechanism of ergosterol peroxide from Paecilomyces cicadae fermentation broth. Int J Mol Sci 2018;19:3935. https://doi. org/10.3390/ijms19123935

2. Ukai S, Kiho T, Hara C, et al. Polysaccharides in fungi. XIII. Antitumor activity of various polysaccharides isolated from Dictyophora indusiata, Ganoderma japonicum, Cordyceps cicadae, Auricularia auricula-judae, and Auricularia species. Chem Pharm Bull 1983;31:741-4. https://doi.org/10.1248/ cpb.31.741

3. Weng SC, Chou CJ, Lin LC, Tsai WJ, Kuo YC. Immunomodulatory functions of extracts from the Chinese medicinal fungus Cordyceps cicadae. J Ethnopharmacol 2002;83:79-85. https://doi.org/10.1016/S0378-8741(02)00212-X

4. Olatunji OJ, Feng Y, Olatunji OO, et al. Polysaccharides purified from Cordyceps cicadae protects PC12 cells against glutamateinduced oxidative damage. Carbohydr Polym 2016;153:18795. https://doi.org/10.1016/j.carbpol.2016.06.108

5. Zeng WB, Yu H, Ge F, et al. Distribution of nucleosides in populations of Cordyceps cicadae. Molecules 2014;19:612341. https://doi.org/10.3390/molecules19056123

6. Wang J, Zhang DM, Jia JF, et al. Cyclodepsipeptides from the ascocarps and insect-body portions of fungus Cordyceps cicadae. Fitoterapia 2014;97:23-7. https://doi.org/10.1016/ j.fitote.2014.05.010

7. Liu SZ, Li XL, Tu GZ, et al. Effects of waste cultured medium of Isaria cicadae on immune factor and growth performance of broilers. China Poult 2012;34:64-5 (in Chinese). https://doi. org/10.3969/j.issn.1004-6364.2012.09.018

8. Zhao LL, Zhi YE, Chen YC, et al. Effect of cordyceps powder feed additive on carcass traits and meat quality in barred rock chicken. J Shanghai Jiaotong Uni (Agricultural Science) 2013; 31:19-22 (in Chinese). https://doi.org/10.3969/J.ISSN.16719964.2013.04.004

9. Yu S, Chai XY, Fan MZ. Analysis of nutritional and bioactive 
components of solid-state fermented substrate of isaria cicadae. Food Machinery 2015;31:155-8 (in Chinese). https://doi.org/ 10.13652/j.issn.1003-5788.2015.01.036

10. Albalasmeh AA, Berhe AA, Ghezzehei TA. A new method for rapid determination of carbohydrate and total carbon concentrations using UV spectrophotometry. Carbohydr Polym 2013;97:253-61. https://doi.org/10.1016/j.carbpol.2013. 04.072

11. Van Soest PJ, Robertson JB, Lewis BA. Methods for dietary fiber, neutral detergent fiber, and nonstarch polysaccharides in relation to animal nutrition. J Dairy Sci 1991;74:3583-97. https://doi.org/10.3168/jds.S0022-0302(91)78551-2

12. Aaslyng MD, Bejerholm C, Ertbjerg P, Bertram HC, Andersen $\mathrm{HJ}$. Cooking loss and juiciness of pork in relation to raw meat quality and cooking procedure. Food Qual Prefer 2003;14:27788. https://doi.org/10.1016/S0950-3293(02)00086-1

13.Straadt IK, Rasmussen M, Andersen HJ, Bertram HC. Aginginduced changes in microstructure and water distribution in fresh and cooked pork in relation to water-holding capacity and cooking loss-A combined confocal laser scanning microscopy (CLSM) and low-field nuclear magnetic resonance relaxation study. Meat Sci 2007;75:687-95. https://doi.org/10. 1016/j.meatsci.2006.09.019

14. Qin C, Huang P, Qiu K, et al. Influences of dietary protein sources and crude protein levels on intracellular free amino acid profile in the longissimus dorsi muscle of finishing gilts. J Anim Sci Biotechnol 2015;6:52. https://doi.org/10.1186/ s40104-015-0052-x

15. Wang H, Liu YM, Qi ZM, et al. An overview on natural polysaccharides with antioxidant properties. Curr Med Chem 2013;20:2899-913. https://doi.org/10.2174/09298673113202 30006

16. Wang Y, Yin H, Lv X, Wang Y, Gao H, Wang M. Protection of chronic renal failure by a polysaccharide from Cordyceps sinensis. Fitoterapia 2010;81:397-402. https://doi.org/10.1016/ j.fitote.2009.11.008

17.Li SP, Zhao KJ, Ji ZN, et al. A polysaccharide isolated from Cordyceps sinensis, a traditional Chinese medicine, protects PC12 cells against hydrogen peroxide-induced injury. Life Sci 2003;73:2503-13. https://doi.org/10.1016/S0024-3205(03) 00652-0

18. Shen L, Lei H, Zhang S, et al. The comparison of energy metabolism and meat quality among three pig breeds. Anim Sci J 2014;85:770-9. https://doi.org/10.1111/asj.12207

19. Cunningham KG, Hutchinson SA, Manson W, Spring FS. Cordycepin, a metabolic product from cultures of Cordyceps militaris (Linn.) link. Part 1. Isolation and characterisation. J Chem Soc 1951;2:2299-300. https://doi.org/10.1039/JR951 0002299

20.Zhang SS, Zhang DS, Zhu TJ, Chen XY. A pharmacological analysis of the amino acid components of Cordyceps sinensis Sacc. Acta Pharm Sin B 1991;26:326-30 (in Chinese).
21.Won KJ, Lee SC, Lee CK, et al. Cordycepin attenuates neointimal formation by inhibiting reactive oxygen species-mediated responses in vascular smooth muscle cells in rats. J Pharmacol Sci 2009;109:403-12. https://doi.org/10.1254/jphs.08308FP

22. Ramesh T, Yoo SK, Kim SW, et al. Cordycepin (3'-deoxyadenosine) attenuates age-related oxidative stress and ameliorates antioxidant capacity in rats. Exp Gerontol 2012;47:979-87. https://doi.org/10.1016/j.exger.2012.09.003

23. Cheng Z, He W, Zhou X, et al. Cordycepin protects against cerebral ischemia/reperfusion injury in vivo and in vitro. Eur J Pharmacol 2011;664:20-8. https://doi.org/10.1016/j.ejphar. 2011.04.052

24. Miyazaki T, Yadomae T, Terui T, Yamada H, Kikuchi T. Studies on fungal polysaccharide XVII. A new glucuronan "protuberic acid" produced by a fungus Kobayashi Nipponica. Biochim Biophys Acta Gen Subj 1975;385:345-53. https://doi.org/10. 1016/0304-4165(75)90363-3

25. Yamada H, Kawaguchi N, Ohmori T, Takeshita Y, Taneya S, Miyazaki T. Structure and antitumor activity of an alkali-soluble polysaccharide from Cordyceps ophioglossoides. Carbohydr Res 1984;125:107-15. https://doi.org/10.1016/0008-6215(84) 85146-0

26. Jiang ZY, Jiang SQ, Lin YC, Xi PB, Yu DQ, Wu TX. Effects of soybean isoflavone on growth performance, meat quality, and antioxidation in male broilers. Poult Sci 2007;86:135662. https://doi.org/10.1093/ps/86.7.1356

27.Zhan XA, Wang M, Zhao RQ, Li WF, Xu ZR. Effects of different selenium source on selenium distribution, loin quality and antioxidant status in finishing pigs. Anim Feed Sci Technol 2007;132:202-11. https://doi.org/10.1016/j.anifeedsci.2006. 03.020

28. Won KJ, Lee SC, Lee CK, et al. Cordycepin attenuates neointimal formation by inhibiting reactive oxygen species-mediated responses in vascular smooth muscle cells in rats. J Pharmacol Sci 2009;109:403-12. https://doi.org/10.1254/jphs.08308FP

29.Zhang C, Luo J, Yu B, et al. Dietary resveratrol supplementation improves meat quality of finishing pigs through changing muscle fiber characteristics and antioxidative status. Meat Sci 2015;102:15-21. https://doi.org/10.1016/j.meatsci.2014. 11.014

30. Monahan FJ, Gray JI, Asghar A, et al. Influence of diet on lipid oxidation and membrane structure in porcine muscle microsomes. J Agric Food Chem 1994;42:59-63. https://doi. org/10.1021/jf00037a009

31.Leandro CG, Ribeiro WS, Santos JAD, et al. Moderate physical training attenuates muscle-specific effects on fibre type composition in adult rats submitted to a perinatal maternal lowprotein diet. Eur J Nutr 2012;51:807-15. https://doi.org/10. 1007/s00394-011-0259-3

32.Staron RS, Malicky ES, Leonardi MJ, Falkel JE, Hagerman FC, Dudley GA. Muscle hypertrophy and fast fiber type conversions in heavy resistance-trained women. Eur J Appl 
Physiol Occup Physiol 1990;60:71-9. https://doi.org/10.1007/ BF00572189

33. Quevedo R, Valencia E, Cuevas G, Ronceros B, Pedreschi F, Bastías JM. Color changes in the surface of fresh cut meat: a fractal kinetic application. Food Res Int 2013;54:1430-6. https:// doi.org/10.1016/j.foodres.2013.10.006

34. Wang L, Wang G, Zhang J, et al. Extraction optimization and antioxidant activity of intracellular selenium polysaccharide by Cordyceps sinensis SU-02. Carbohydr Polym 2011;86: 1745-50. https://doi.org/10.1016/j.carbpol.2011.07.007

35. Gong M, Zhu Q, Wang T, Wang XL, Ma JX, Zhang WJ. Molecular structure and immunoactivity of the polysaccharide from Cordyceps sinensis (Berk) Sacc. Sheng Wu Hua Hsueh Tsa Chih 1990;6:486-92 (in Chinese).

36. Chen WX, Zhang WY, Shen WB, Wang KC. Effects of the acid polysaccharide fraction isolated from a cultivated Cordyceps sinensis on macrophages in vitro. Cell Immunol 2010;262: 69-74. https://doi.org/10.1016/j.cellimm.2010.01.001

37. Chen YJ, Shiao MS, Lee SS, Wang SY. Effect of Cordyceps sinensis on the proliferation and differentiation of human leukemic U937 cells. Life Sci 1997;60:2349-59. https://doi.org/10.1016/ S0024-3205(97)00291-9

38. Sun YF, Sun Y, Wang Z, et al. Isaria cicadae conidia possess antiproliferative and inducing apoptosis properties in gynaecological carcinoma cells. Mycology 2017;8:327-34. https:// doi.org/10.1080/21501203.2017.1386243

39.Zeng Y, Lian S, Li D, et al. Anti-hepatocarcinoma effect of cordycepin against NDEA-induced hepatocellular carcinomas via the PI3K/Akt/mTOR and Nrf2/HO-1/NF- $\kappa B$ pathway in mice. Biomed Pharmacother 2017;95:1868-75. https://doi. org/10.1016/j.biopha.2017.09.069

40. Weber A, Wasiliew P, Kracht M. Interleukin-1 (IL-1) pathway. Sci Signal 2010;3:cm1. https://doi.org/10.1126/scisignal.3105 $\mathrm{cm} 1$

41. Granowitz EV, Clark BD, Vannier E, Callahan MV, Dinarello CA. Effect of interleukin-1 (IL-1) blockade on cytokine synthesis: I. IL-1 receptor antagonist inhibits IL-1-induced cytokine synthesis and blocks the binding of IL-1 to its type II receptor on human monocytes. Blood 1992;79:2356-63.

42. Netea MG, Nold-Petry CA, Nold MF, et al. Differential requirement for the activation of the inflammasome for processing and release of IL-1beta in monocytes and macrophages. Blood 2009;113:2324-35. https://doi.org/10.1182/blood-2008-03146720

43. Hoffmann E, Thiefes A, Buhrow D, et al. MEK1-dependent delayed expression of fos-related antigen- 1 counteracts c-Fos and p65 NF- $\kappa \mathrm{B}$-mediated interleukin- 8 transcription in response to cytokines or growth factors. J Biol Chem 2005;280: 9706-18. https://doi.org/10.1074/jbc.M407071200

44. Gaestel M, Kotlyarov A, Kracht M. Targeting innate immunity protein kinase signalling in inflammation. Nat Rev Drug Discov 2009;8:480-99. https://doi.org/10.1038/nrd2829

45. Dinarello CA. Immunological and inflammatory functions of the interleukin-1 family. Annu Rev Immunol 2009;27:51950. https://doi.org/10.1146/annurev.immunol.021908.132612

46. Martinon F, Mayor A, Tschopp J. The inflammasomes: Guardians of the body. Annu Rev Immunol 2009;27:229-65. https:// doi.org/10.1146/annurev.immunol.021908.132715 